\title{
Estimate Supply Chain robustness using asymmetric loss functions
}

\author{
Luca Canetta \\ Department of Innovative \\ Technologies (DTI) \\ SUPSI \\ Manno, Switzerland \\ luca.canetta@supsi.ch
}

\author{
Naoufel Cheikhrouhou \\ Haute école de gestion de Genève, \\ HES-SO University of Applied \\ Sciences Western Switzerland, \\ CH-1227, Geneva, Switzerland
}

\author{
Rémy Glardon \\ Ecole Polytechnique Fédérale de \\ Lausanne (EPFL) \\ Lausanne, Switzerland
}

\begin{abstract}
Supply Chains have to be designed and managed holding simultaneously into account many different performance measures. Moreover, modern Supply Chains have to ensure satisfying performances despite an increasing degree of complexity and market uncertainty as well as be capable to limit the negative impacts of disruptive events. A multi-criteria robustness evaluation framework is proposed to deal with these challenges. The proposed approach allows to separately assessing the impact of various performance measures specifying tailor loss functions, being able to deal with non-linearity and asymmetric impacts. Moreover, an original Robustness Index is defined, in order to provide reliable estimations even in the presence of outliers and integrating information about kurtosis and skewness in the robustness estimation. The proposed framework is applied to a fictive industrial case to demonstrate its utilization and show the kind of analysis that can be done on the basis of the obtained results. The approach, simply requiring the definition of some parameters and the description of the characteristics of the Supply Chain configurations to be evaluated, is meant to be easily used by practitioners.
\end{abstract}

Keywords-multi-criteria analysis; robustness; loss-function; simulation; Supply Chain

\section{INTRODUCTION}

Supply Chain (SC) design and management is becoming increasingly complex due to, on the one hand, the proliferation of Key Performance Indicators (KPIs) required for dealing with economic, social and environmental performances to be satisfied, and, on the other hand, to the shift towards a more comprehensive and long term oriented assessment framework. This implies the development of comprehensive evaluation frameworks covering multivariate performance measurement and allowing to perform various types of analysis, integrating scenario analysis and stochastic elements. In fact, efficiency is not anymore the only aspect to consider because concepts like risk management, resilience and robustness are becoming more and more important. Thus, it is necessary evaluate all the aspects of the market environment uncertainty and variability, going from the inner stochastic nature of customer demand to the uneven and occasional occurrence of major disruptions. This results into the development and application of various concepts such as robustness, flexibility and resilience $[1,2,3]$. The different definitions of resilience have a common element.
Enterprise resilience is a response to expected and unforeseen changes, disruptive events and disturbances and it is the ability for a company to adapt, respond and recover from these changes [4]. Flexibility refers to the capacity of a system to adapt itself to a changing environment $[3,5]$. Robustness is the property of a given system to accommodate for factors uncertainty and variability without significant degradation/deviation from the initial desired state $[6,7,8,9$, $10,11,12,13,14,15,16]$. The main difference among these concepts is that resilience and flexibility imply that the system configuration is not fixed and can evolve in function of the changing requirements, while robustness assumes a given configuration.

The aim of this work is providing the tools supporting the decision makers during the choice of a robust SC configuration that can ensure satisfying performance in many different market environment scenarios. For this reason, a new robustness index is proposed. First of all, a multi-criteria performance measurement system is developed in order to go further the typical limitations of cost based performance evaluation [17]. This implies to specify for each KPI a mathematical expression linking its value with its contribution to the overall suitability of a given SC. This is done using specifically developed asymmetric loss (respectively utility) functions in order to easily handle the inner non linearity of SC behaviour. The elaborated overall loss function is used to estimate the SC performance in each of the potential scenarios and constitutes the main input for the calculation of the proposed robustness index.

The application of the proposed robustness evaluation framework is illustrated analyzing the behavior of a fictive production system, which behavior is assessed through a discrete event simulation model in order to appropriately take into account the stochastic nature of SC processes. The robustness is evaluated over a long time horizon focusing on the evolution of various customer demand scenarios, which result from the definition of various sales channels strategies. For a comprehensive description of the demand scenarios' characteristics see [18]. In the next section are described the selected KPIs and the suitable Loss Functions (LFs). In section 3 are defined the Robustness Index (RI) and the robustness evaluation framework. In section 4 are summarized the results 
obtained applying the proposed approach to a fictive case, in order to determine the most suitable SC configuration among 15 alternatives considering the trade-off between efficiency and robustness. Finally, section 5 provides the conclusions and outlines future research perspectives.

\section{KPIS AND LOSS FUNCTION IDENTIFICATION}

The proposed robustness evaluation framework can be applied to any number of quantitative KPIs. In this work for simplicity the focus is restrained to some economic KPIs dealing with well-known production management issues. An extension to social and environmental KPIs can be found in [19]. The use of quantitative KPIs avoids the problems related to the use of qualitative indexes. In fact, the latter implies the recourse to subjective evaluations, which can be difficult to establish and for their inner nature can be questionable, raising validity issues for the definition of suitable LFs and thus for the entire framework.

The considered aspects are customer satisfaction, inventory level and equipment utilization. These widely used KPIs have been selected in order to facilitate the comparison among various industrial sectors and for because of for them are available widely known benchmarks, which existence simplifies the identification and parameterization of the loss functions. The KPIs are used for measuring the performance achieved by a given SC configuration for each demand scenario. The LFs are computed on the basis of the deviation of the actual value of a KPI from a Reference Value, which represents a satisfying performance level.

\section{A. Customer satisfaction}

The Service Level (SL) describes the capacity of a system to deliver the required product/services to its customer within a previously established lead time. It is thus a measure of customer responsiveness and it constitutes one of the drivers of customer satisfaction. Various SL definitions are typically employed in practice. In this work the SL is defined as the percentage of orders that are fulfilled on-time. On-time fulfillment means the instantaneous availability of on-hand inventory in a Make-To-Stock (MTS) environment, while in a Make-To-Order (MTO) or in an Assembly-To-Order (ATO) context implies to deliver the goods/services to the customer within a pre-specified lead time.

$$
S L=\frac{N O_{O T}}{N O}
$$

\section{where $\quad \mathrm{NO}_{\text {OT }} \quad$ Number of orders fulfilled on-time

$$
\text { NO Number of customer received orders }
$$

Usually the impact of SL on customer satisfaction is characterised by a nonlinear relationship [20, 21, 22, 23]. Thus, the suitable LF has to be nonlinear and also asymmetric. In the case of an asymmetric LF both the sign and the magnitude of the SL deviation from the satisfying SL reference value $\left(S_{R V}\right)$ influence the resulting loss value. The use of asymmetric LFs, for instance Linex and BLinex, has been already recommended while evaluating aspects related to the interaction with customers and people in general [21, 24, 25, 26, 27, 28]
A new LF, called the Satisfaction Bounded Linex (SBLinex), is proposed in order to overcome the BLinex Taguchi "nominal the best" approach, which it is not suitable for SL analysis. The parameterisation of the SBLinex LF is influenced by the necessity to apply an asymmetric approach, where SL degradations are penalised more than are rewarded equivalent SL enhancements. The value of the SBLinex LF is computed according to (2).

$$
L_{S B L i n e x}(S L)=-\operatorname{sign}\left(S L-S L_{R V}\right) \cdot \frac{1}{\lambda}\left[1-\frac{1}{1+\lambda \cdot b \cdot\left[e^{a\left(S L-S L_{R V}\right)}-a \cdot\left(S L-S L_{R V}\right)-1\right]}\right]
$$

$\begin{array}{cll}\text { where } \quad \operatorname{sign}\left(S L-S L_{R V}\right) & =-1 \quad \text { for } \quad \mathrm{SL}<\mathrm{SLt} \\ & =0 \quad \text { for } \quad \mathrm{SL}=\mathrm{SLt} \\ & =1 \quad \text { for } \quad \mathrm{SL}>\mathrm{SLt} \\ & & \begin{array}{l}\text { bounding parameter (the loss is } \\ \text { bounded between } 0 \text { and } 1 / \lambda\end{array} \\ & \\ b>0 & \text { asymmetry parameter } \\ \mathrm{a}<0 & \text { scale parameter (curve flatness) } \\ & \text { and specifying which } S L \\ & \text { deviations are more penalised }\end{array}$

Fig. 1 and Fig. 2 allow to gain an understanding of the influence of SBLinex parameters on the resulting loss values and its ability to represent correctly the impact of SL degradation.

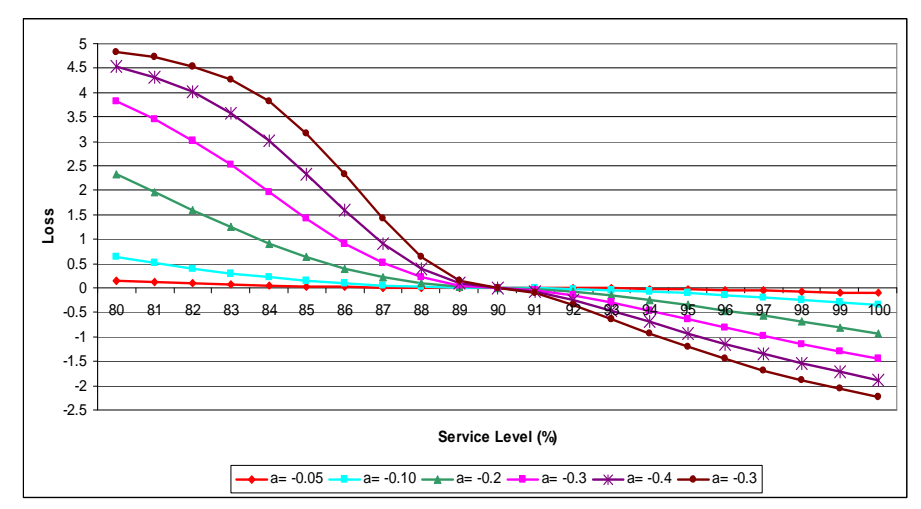

Fig. 1. SBLinex $(\lambda=0.2, b=1$ and $S L R V=90 \%)$

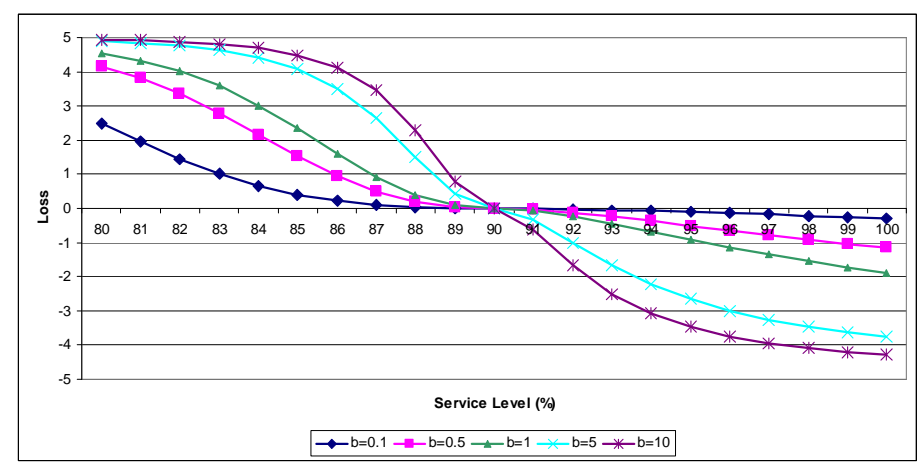

Fig. 2. SBLinex $(\lambda=0.2, a=-0.4$ and $\operatorname{SLRV}=90 \%)$ 


\section{B. Inventory Level}

Inventory related measures are typically applied for production system performance analysis [29]. The Inventory Turnover Ratio (ITR) is selected as inventory related KPI in order to ensure an easy comparison of the results obtained by different production systems as well as to facilitate the establishment of reliable inventory level thresholds. ITR is a suitable inventory KPI because it establishes a relationship between the inventory level and the demand level (3). The availability of ITR data facilitates the estimation of industrial sector benchmarks that can be used as first approximation of the inventory level reference value required for the loss calculation.

$$
I T R=\frac{\text { Demand }}{I L}
$$

where Demand Overall demand over a specific time horizon (usually one year)

\section{IL Average Inventory Level}

An asymmetric LF is implemented for measuring the loss induced by the deviation of the ITR from the established reference value. An increase of the ITR is beneficial because it is linked to an inventory level decrease and results into a negative loss value. On the other hand, an ITR decrease is associated with a positive loss, because it implies an inventory level increase with the relative additional costs. Thus, in this case a "the greater the better" Taguchi approach is followed. Making the hypothesis that the modification of ITR values only influences the amount of inventory carrying costs, it is possible applying the asymmetric linear based LF, called LIN-LIN [24, $26,30,31,32,33$ ] The broad applicability of LIN-LIN LF is further confirmed by the fact that this LF can be used to approximate other asymmetric LF [33]. The proposed LIN-LIN LF (4) specifies the loss value on the basis of the deviation from a benchmark, called ITR reference value $\left(I_{T R}\right)$. The latter can be fixed looking at the performance of the top class companies belonging to the same industrial sector or, if the focus is mainly the company under analysis, it can be elaborated starting from the current company performance in terms of inventory management.

$$
L_{L I N-L I N}(I T R)=\left\{\begin{array}{lll}
-d \cdot\left(I T R-I T R_{R V}\right) & \text { if } & I T R<I T R_{R V} \\
-e \cdot\left(I T R-I T R_{R V}\right) & \text { if } & I T R>I T R_{R V}
\end{array}\right.
$$

where $\mathrm{d}, \mathrm{e}>0$ the loss magnitude is greater for ITR $\mathrm{d}>\mathrm{e} \quad$ degradations than for ITR enhancements

In the framework of the asymmetric LIN-LIN LF the choice of penalising more stock increase than rewarding stock reduction is justified by the fact that an ITR increase, thus a stock decrease, directly implies only a decrease of the financial component of the inventory carrying costs. On the other hand, the decrease of the ITR, which results into a stock increase, provokes an increase of all the variable components of inventory carrying costs.

\section{Resource utilisation}

A straightforward measure of resource utilisation is the workload, calculated as "the ratio of the direct time charged for production activities (setup and processing time) to the clock time scheduled to be available for a given period of time" [29]. However, the information that can be gathered from workload analysis provides a too aggregated vision of the resource utilisation. For this reason, this work proposes another KPI related to resource utilisation, which clearly assesses the impact of setup time and processing time. The Setup Time Percentage (SUT\%), calculated according to (5), can be correlated with the total production cost. Furthermore, given a fixed production system infrastructure and a specified constant production volume, measuring the SUT\% can allow to monitor and to explain the workload variations induced by the use of different production planning and control strategies.

$$
S U T \%=\frac{\sum_{i=1}^{n} S U T_{i}}{\sum_{i=1}^{n} S U T_{i}+\sum_{i=1}^{n} O P T_{i}}=\frac{\sum_{i=1}^{n} S U T_{i}}{\sum_{i=1}^{n} S U T_{i}+U P T \sum_{i=1}^{n} O Q_{i}}
$$

where

$\mathrm{n}$ number of fulfilled order

$\mathrm{SUT}_{\mathrm{i}} \quad$ Setup time for order $i$

$\mathrm{OPT}_{\mathrm{i}}$ Order Processing Time for order $i$

UPT Unitary Processing Time ( constant)

$\mathrm{OQ}_{\mathrm{i}}$ Order Quantity for order $i$ (the pattern of the OQ is strongly dependent from manufacturing constraints and the chosen PP\&C strategy)

A simple linear loss function (LIN), defined in (6), is used to quantify the impact of the deviations from the SUT\% reference value $\left(\mathrm{SUT} \%_{\mathrm{RV}}\right)$. The use of a linear loss function to approximate the influence of SUT\% is derived from a "costbased" estimation. The SUT\% $\%_{\mathrm{RV}}$ mainly reflects the specificities of the production system under analysis and can thus be fixed equal to the currently achieved SUT\%, unless the performance of the production system is considered not satisfying.

$$
\begin{array}{ccl} 
& \multicolumn{5}{c}{L_{\text {LIN }}(S U T \%)=} & f \cdot\left(S U T \%-S U T \%_{R V}\right) \\
\text { where } \quad \mathrm{f}>0 \quad & \begin{array}{l}
\text { coefficient controlling the loss } \\
\text { magnitude }
\end{array}
\end{array}
$$

The coefficient $f$ has to be defined taking into account the criticality and the cost associated to the production resources. The value of $f$ is also influenced by the workload level, if the production resource utilisation is close to saturation the negative impact of an increased SUT\% is magnified, because it can increase the queuing time.

\section{Loss discounting factor}

In this work each scenario is evaluated over a time horizon spanning various years. In order to give a greater importance to the performance achieved in the periods closer to the present, 
the application of a discounting factor to the loss values is proposed (7). The use of discounting factor is common practice in multi-period cost evaluation and has been already introduced in robustness analysis [34].

$$
\operatorname{Loss}_{j}(i)=\frac{1}{H o r} \cdot \sum_{t=1}^{H o r} \frac{\operatorname{Loss}_{j}(i, t)}{(1+r)^{t-1}}
$$

where

Hor number of periods included in the considered time horizon

$\operatorname{Loss}_{j}(i, t) \quad$ loss value of KPI $j$ for scenario $i$ and time period $t$

$\operatorname{Loss}_{j}(i) \quad$ average loss value of KPI $j$ for scenario $i$ considering the entire time horizon (Hor)

$$
j=\{S L, I T R, S U T \%\}
$$

$r$ discounting factor

The choice of a suitable value of $r$ has to be done on the basis of the decision maker risk aversion.

\section{E. Overall loss}

The losses, calculated on the basis of the values actually achieved by the various KPIs and of the established reference values, provide the foundation for developing a robustness evaluation tool, which aims at supporting the decision makers for the identification of the critical SC configuration parameters. Due to the concurrent recourse to various KPIs, two alternatives are available for the performance as well as for the robustness evaluation.

The first alternative relies upon the definition of definition of a vector of losses, which allows to manage simultaneously the various KPIs and to keep them distinct. These vectors can be compared in order to obtain a Pareto frontier [34, 35], constituted by the non-dominated solutions, which is represented by a hyper plan of cardinality equivalent to the number of implemented KPIs. This approach allows to compare the scenarios preserving their diversity without being obliged to establish from the beginning trade-off among them. However, this approach is extremely time consuming and due to the difficulty to discriminate many non-dominated solutions especially in high dimensional space and to the highly subjectivity of this phase, the quality of the final ranking can be unsatisfying when dealing with real-world applications.

The second alternative transforms the multi-criteria KPI vector into a unique mono-criteria index establishing trade-off among the various KPIs usually relying upon a linear additive model, which results in a weighted sum of the various losses $[13,36,37,38]$. This approach allows a fast calculation and can be simply integrated in many tools. This approach requires the explicit specification of the decision maker preferences and the establishment of trade-offs among the various KPIs, which are then translated into weighting parameters. The recourse to a unique index, implies the risk of losing information about the solution diversity, thus about the contribution of each loss for a specific case. The strong dependency of the final ranking by the weighting results can raise some concerns about the validity of the weights' estimation, the latter has to rely upon formalised and structured approaches in order to increase the confidence about the obtained results. For instance, the Analytical Hierarchical Process (AHP), which is based on pairwise comparison among the KPIs, is an interesting tool for weight estimation in the framework of robustness analysis [13, 36] and multi-criteria decision making [37, 39].

In this work, in order to simplify the decision maker task and to automate as much as possible the various steps of the robustness evaluation framework, the second approach is chosen. Thus a unique index, called Overall Loss (OL), is calculated applying a linear additive model as shown in (8). The relative importance of the various performances is established by the decision maker defining some weighting coefficients $\left(w_{j}\right)$. The weighting coefficients also play a role in scaling the contributions of losses defined over various range of values in order to assess that all KPIs are effectively taken into account in the performance evaluation.

$$
\begin{aligned}
& O L(i)=\sum_{j=1}^{J} w_{j} \cdot \operatorname{Loss}_{j}(i) \\
& \sum_{j=1}^{J} w_{j}=1 \\
& w_{j} \geq 0
\end{aligned}
$$

where

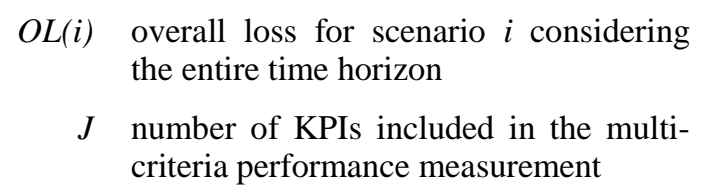

In the application case described in section IV, in order to deal with three KPIs the weighting factors defined in Table II have been applied.

\section{ROBUSTNESS DEFINITION AND ASSESSMENT}

The interest for SC robustness is continuously increasing as proven by the results contained in [3], which show how the number of publications dealing with the subject is significantly increasing from 2001. However, an unambiguous robustness definition does not exist yet. Furthermore, some of these definitions are at least partially overlapping with other concepts, being a merger of robustness, flexibility and resilience concepts $[2,3,5,13]$.

A first classification concerning robustness definitions in the context of production management focuses on the way in which the insensitivity of a solution with respect to input variability is estimated. Quality robustness is analysed focusing on the objective function space and thus estimating performance variability. A given (fixed) SC configuration is considered as "quality robust" if the performance remains at satisfying levels while the company environment is varying. Quality robustness is widely applied in production management $[3,5,6,7,9,11,12,15,40,41]$ and in other contexts $[10,14,42]$. On the other hand, solution robustness is estimated analysing the solution space. A SC is "solution robust" if the configurations achieving the best performance for 
the various market environments scenarios to be considered are similar to the baseline configuration. Thus, solution robustness is closely related to flexibility.

In the following the analysis will be concentrated on quality robustness. Quality robustness is usually estimated focusing on input data that are external to the SC configuration design and mainly beyond the control of the SC shareholders. As in this work the company environmental factor that is most commonly used in robustness estimation is customer demand [6, 7, 11, 15]. However, internal and external sources of uncertainty can be simultaneously considered in robustness evaluation [41, 43].

Many robustness definitions exist, these represent different interpretations of the robustness concept also influenced by the considered specific risk attitude. Moreover, many robustness evaluation frameworks and equations are proposed. The latter differentiate themselves also with respect to the quantity and typology of data required for robustness estimation. The robustness definitions (absolute, deviation, relative) proposed in [44] are very conservative, thus reflect a strong risk aversion, because they focus robustness calculation on the worst case performance. The main advantage of the worst case based robustness measures is that they do not require an explicit computation of the probability of existence of the various market environment scenarios. However, not considering the scenario probability of existence is also one of their main drawbacks, because the robustness estimation can be based on a scenario having a little probability of existence and thus not reflecting the overall market environment in which the SC evolves.

In order to take into account the information stemming from all potential market environment scenarios other types of robustness measure have been introduced. For instance, the "compromise robustness" proposed in [13] implies that "a robust solution is one that is satisfactory to the decision maker in as many scenarios as possible without being too unsatisfactory to the decision maker in any single scenario" [13]. This definition takes into account all the scenarios, even if their probability of existence is not required, because the idea is having a production system showing satisfying performance in as many scenarios as possible without being too unsatisfactory in any scenario. Some robustness definitions require that the performance is within an acceptability zone, thus within a range of deviation from optimality that is considered acceptable by the decision maker, for all scenarios or the great majority of scenarios $[9,10]$.

The availability of the probability of occurrence of all scenarios, or in alternative an assumption about this probability distribution, allows to apply robustness probabilistic approaches [43] or approaches based on weighted average, where the importance of the performance degradation obtained in a specific scenario is weighted according to its likelihood of occurrence $[7,11,12,16]$.

\section{A. Robustness index definition}

The characteristics of the robustness index strongly depend on the objectives of the established robustness evaluation framework and on the nature and quantity of data that can be gathered. In this research context the objective is determining the quality robustness of a given SC configuration, which is supposed to remain unchanged, facing significant potential market environment modifications due to the disruptive modification of sales channel strategy (outer robustness). For each scenario is estimated the demand evolution covering 10 years as well as the probability of occurrence, see the details in [45].

The availability of the probability of occurrence of each scenario allows the use of a wide range of robustness measures. In such a context an approach based on weighted average measures provides a better representation about the overall performance of a solution. However, the risk of underestimating the negative effect of an unacceptable poor performance for some scenarios has to be minimized, especially when dealing with outer robustness.

A new Robustness Index (RI) is proposed (9) in order to combine the strengths of the previous robustness definition typologies.

$$
\begin{aligned}
& R I=\frac{1}{\alpha \cdot\left(O L_{25 \%}-O L_{5 \% \%}\right)+\beta \cdot\left(O L_{50 \%}-O L_{5 \% \%}\right)+\gamma \cdot\left(O L_{15 \% 6}-O L_{5 \%}\right)+\delta \cdot\left(O L_{95 \%}-O L_{5 \% \%}\right)} \\
& \text { where } O L_{i} \% \quad \text { OL for } i \text { percentile of cumulated } \\
& \left(O L_{j \%}-O L_{i \psi}\right) \quad O L \text { difference between two } \\
& \text { cumulated probability percentile } \\
& \alpha, \beta, \gamma, \delta \geq 0 \quad \text { weights modulating the impact of } \\
& \alpha+\beta+\gamma+\delta=1 \quad \text { the four } O L \text { differences on the } R I \\
& \alpha \leq \beta \leq \gamma \leq \delta \quad \text { Weights for "big losses" are } \\
& \text { greater (risk adverse decisions) }
\end{aligned}
$$

Fig. 3 illustrates how starting from the OL values achieved by a given SC configuration, in the various scenarios, is possible computing the RI and thus specifying its relative robustness. The probability of occurrence of the various scenarios can be not uniform. First of all, the OL are ordered following an increasing order. This allows to determine for which OL values the cumulated scenario probability of occurrence reach some specific percentile. Five percentile values $(5 \%, 25 \%, 50,75 \%, 95 \%)$ have been chosen in order to provide information about the OL cumulated distribution function.

Considering various percentiles allows to better represent the entire probability distribution pattern, capturing the influence of the third and fourth moments of the probability distribution (skewness and kurtosis). The proposed RI is calculated discarding the loss values below OL5\% and above OL95\%. In this way, the bias due to potential outlier scenarios characterised by extreme loss values is limited. This is done making the hypothesis that these scenarios represent only a negligible cumulated probability of occurrence.

All the differences are measured with respect to OL5\%, the focus on the best performance is quite uncommon in robustness literature, where often the reference points are the worst case performance or the boundaries of the acceptable zone. However, in this way the RI definition well represent the objective of ensuring the closeness to the best performance. The RI equation also facilitates the tailoring, on the basis of decision maker risk aversion, of the weighting coefficients $(\alpha$, 
$\beta, \gamma, \delta$ ) involved in its estimation. The value of weighting these coefficients applied in the specific case of section IV are shown in Table 2. The ranking of SC configuration on the basis of the proposed RI proves to be quite robust against the choice of the weighting parameters. The choice of the weighting coefficients do not alter significantly the robustness ranking of the various SC, allowing to identify the most robust and the least robust configurations. In practice, the choice of a suboptimal weighting mainly shrink the differences among the RI values [45].

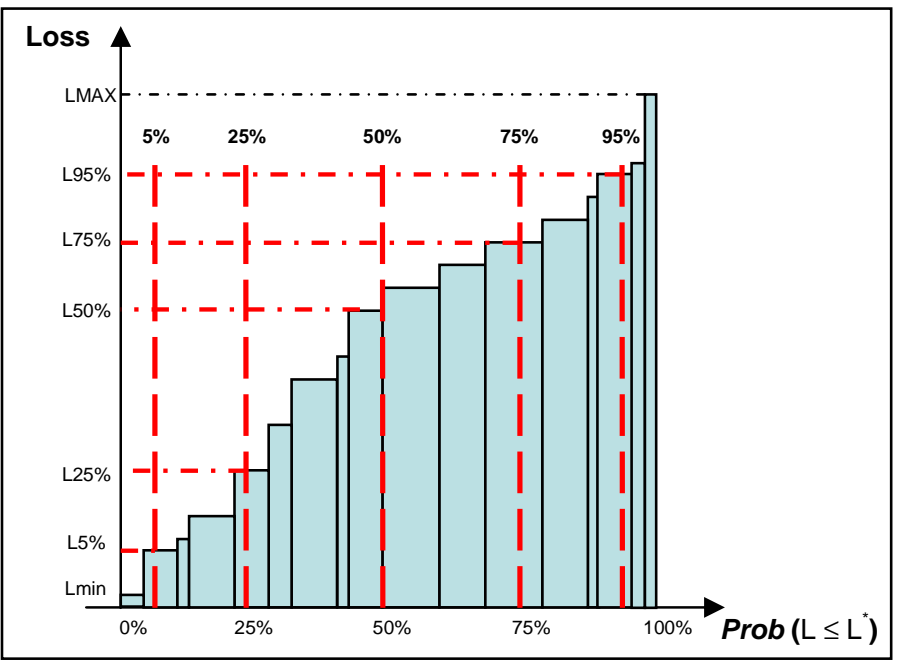

Fig. 3. Illustrative application of the proposed Robustness Index

It must be noticed that RI measures only the variability of the performances. The evaluation of the SC configuration overall suitability requires the use of the proposed RI in association with another index, specifying the expected performance. The Average Overall Loss (AOL) is used for this purpose (10). The OL weighted average is preferred to the OL median, because the former takes into account all losses and is better suited for dealing with potentially skewed probability distributions.

$$
A O L=\sum_{i=1}^{N_{S C E N}} p(i) \cdot O L(i)
$$

$$
\begin{aligned}
& \text { where } \quad N_{S C E N} \quad \text { overall number of scenarios } \\
& p(i) \quad \text { probability of existence scenario } i \\
& O L(i) \quad \text { Overall Loss related to scenario } i \\
& \sum_{i=1}^{N_{S C E N}} p(i)=1 \quad \begin{array}{l}
\text { Because all the considered } \\
\text { scenarios have to be evaluated }
\end{array}
\end{aligned}
$$

The proposed $R I$ is characterized by some interesting features:

- correctly accounting for the impact of performance variability, skewness and kurtosis. Basing the RI estimation on the OL corresponding to five meaningful percentiles allows to capture in a simple and computationally parsimonious manner the shape of the cumulated probability distribution.
- $\quad$ efficacy dealing with potential outlier scenarios. The RI computation, is based on the OL comprised between the $5 \%$ and the $95 \%$ percentile. The considered subset of OL does not contain the most critical and least critical values, decreasing the influence of potential outliers with not significant probability of occurrence.

- the solution ranking based on robustness values is independent from the established values for reference values and other parameters. Using RI is always possible discriminating the most robust $\mathrm{SC}$ configurations from the non-robust ones.

\section{APPLICATION OF THE ROBUSTNESS EVALUATION FRAMEWORK TO A SPECIFIC CASE}

In this section, all the steps of the proposed robustness evaluation framework are applied to numerical data in order to clarify the followed approach and the kind of analysis that can be undertaken. 28 demand scenarios are generated to estimate the performance of $15 \mathrm{SC}$ configurations. In this application, the number of scenarios and SC configuration is intentionally kept low in order to propose an example of manageable size.A discrete event simulation model is applied to estimate the behaviour of the various configurations accurately taking into account the stochastic nature of the market demand and of the production processes. The detailed description of the various demand scenarios and SC configurations can be found in [45].

The 15 SC configurations are obtained modifying some inventory management parameters of the original SC configuration. For doing that Central Composite Design (CCD) is selected, it is a well-known Design Of Experiments already used in robustness evaluation [41]. The value of the parameters of the original SC configuration have been respectively decreased and increased of $20 \%$. A modification of $20 \%$ is chosen because it is expected that can produce significant performance modifications without completely changing the SC behavior. CCD has also been chosen because it allows to estimate quadratic Response Surface Models (RSMs). The application of RSM or other meta-modelling techniques, in order to gain qualitative and quantitative insight about the relationship linking RI and AOL values to those of the SC parameters, is the natural following step of robustness assessment. The recourse to meta-modelling techniques is particularly suitable for real industrial applications, where the high number of involved factors makes almost impossible to extract valuable information from the direct analysis of simulation results.

In order to apply the robustness evaluation approach, have to be specified the parameters related to the specific LFs and the weighting factors for OL and RI, which are provided in table I and II. The weighting coefficients and the discounting factor, which is fixed to $\mathrm{r}=0.1$, are specified according to the decision maker attitude and risk aversion and can be directly specified by the user or inferred using decision support tools like AHP. 
TABLE I. LF PARAMETERS FOR SL, ITR AND SUT\%

\begin{tabular}{|l|c|c|c|c|c|c|}
\hline \multicolumn{1}{|c|}{ KPI } & \multicolumn{2}{c|}{ SL } & \multicolumn{2}{c|}{ ITR } & SUT\% \\
\hline Reference Value & \multicolumn{3}{|c|}{$90 \%$} & \multicolumn{2}{c|}{70} & $42 \%$ \\
\hline Loss Function & \multicolumn{2}{c|}{ SBLINEX } & \multicolumn{2}{c|}{ LIN-LIN } & LIN \\
\hline LF Parameters & $\mathrm{a}$ & $\mathrm{b}$ & $\lambda$ & $\mathrm{d}$ & $\mathrm{e}$ & $\mathrm{f}$ \\
\hline Assigned values & -0.5 & 10 & 0.01 & 0.25 & 0.125 & 0.5 \\
\hline
\end{tabular}

TABLE II. WEIGHTING FACTORS FOR OL AND RI ESTIMATION

\begin{tabular}{|c|c|c|c|c|c|c|}
\hline \multicolumn{3}{|c|}{$\begin{array}{c}\text { Overall Loss weighting } \\
\text { coefficients }\end{array}$} & \multicolumn{4}{c|}{$\begin{array}{c}\text { Robustness Index weighting } \\
\text { coefficient }\end{array}$} \\
\hline$w S L$ & $w I T R$ & $w S U$ & $\alpha$ & $\beta$ & $\gamma$ & $\boldsymbol{\beta}$ \\
\hline 0.4 & 0.3 & 0.3 & 0.1 & 0.2 & 0.3 & 0.4 \\
\hline
\end{tabular}

As already specified, the values of these parameters have only a relative influence on the obtained results and thus can be chosen also making reference to the literature or applying simple decision making rules.

The use of RI and AOL for ranking various production system configurations is investigated. In this way, the benefits of the proposed robustness evaluation framework can be easily assessed. As can be remarked in Fig. 4 the behaviour of the various SC configurations differs significantly. In particular, the following configurations show completely unsatisfying values of AOL: 2, 3, 4, 5, 10 and 14. This is due to a drastic degradation of SL, which results into high values of loss due to the use of a SBLinex loss function. On the other hand, another group of configurations $(1,6,7,8,11$ and 12) show satisfying results both in terms of AOL and RI (Fig. 5). These configurations achieve in comparison with the reference SC (configuration 15) better results both in terms of AOL and RI. This simple application demonstrates that the proposed robustness evaluation framework allows to determine the SC critical factors and to support the choice of a suitable configuration. It is interesting noticing that the SC configurations achieving the best results are not necessarily similar. This suggests that the interaction among the various parameters plays an important role and thus confirms the complex and extreme interrelated nature of the production system.

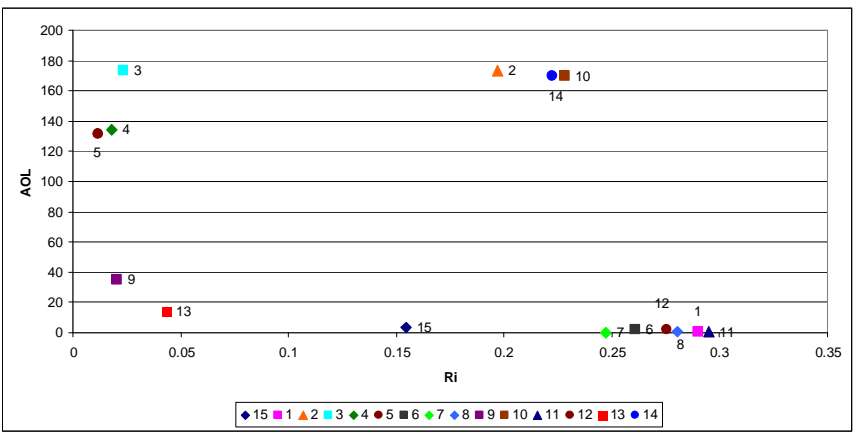

Fig. 4. AOL and RI characterisation of the 15 production system configurations

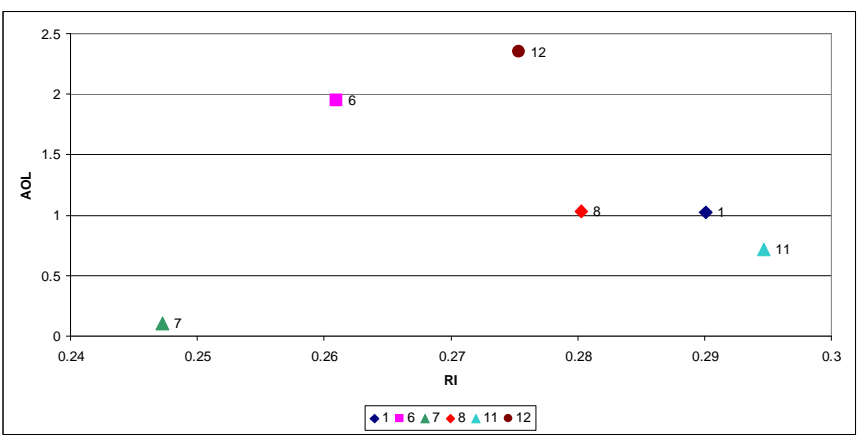

Fig. 5. AOL and RI characterisation for the most promising production system configurations

In this illustrative case, RSM is applied in order to estimate the influence of the considered design factors. On the basis of the available data, the parameters of various models can be estimated: linear, linear with interactions, pure quadratic, and full quadratic. The possibility to test various additive models allows to better discriminate the importance of the various design factors. The estimated parameter of two full quadratic models, those using a complete second order polynomial regression model, one for RI and the other for AOL, are provided in order to determine the impact of the various design factors on each performance measure (Table III). It can be remarked as for both RI and AOL the only statistically significant factor is b1, which describes the constituted by the inventory management parameters for a specific subassembly, namely subassembly A. Due to the arbitrary selection of the parameters and of the levels to be included in the DOE for this application, as well as to the fact that not all the parameters have been tested, the regression analysis has to be interpreted circumspectly and the obtained indications have to be further validated with more comprehensive studies. Nevertheless, the indications about the statistical significance of b1 and the sign of this regression coefficient seem confirmed looking at the various SC configurations. Considering RI the four most satisfying configurations (number 11, 1, 8 and 12) are characterised by the lowest level of the inventory management parameter of subassembly A, as suggested in the RSM model by the negative value of $b 1$ coefficient. The positive value of b1 coefficient for AOL model implies that the most satisfying production configurations are also characterised by the lowest level of the inventory management parameter of subassembly $\mathrm{A}$; this is the case for the four most satisfying configuration in terms of AOL (number 7, 11, 1,8).

Better regression results can be obtained analyzing more SC configuration parameters as well as applying variable transformation. However, in this phase these refinements are not considered necessary because the primary objective of this application is simply providing an explanation of the use of meta-modelling techniques for analyisng the data concerning $\mathrm{RI}$ and $\mathrm{AOL}$ and not furnishing the best parameter estimations to be subsequently used in a real industrial application. 


\section{CONCLUSIONS}

The proposed framework covers the robustness evaluation process, starting from the choice of a multi-criteria performance metric to arrive to the definition of a new robustness index and to the development of the tools required for its computation. The simultaneous use of various loss functions to describe the impact of KPIs deviations from targets as well as the recourse to asymmetric loss functions constitute a refinement of the traditional performance estimation method and allows to better take into account the trade-offs among the various KPIs also during the robustness estimation.

The defined RI has the advantage of fully exploiting the information about the probability of occurrence of the various scenarios, when they are available, and thus to integrate in the robustness calculation a reach description of the scenario distribution pattern.

TABLE III. PARAMETER ESTIMATION OF RSM FULL QUADRATIC MODELS FOR RI AND AOL

\begin{tabular}{|c|c|c|c|c|c|c|c|c|}
\hline & \multicolumn{4}{|c|}{ RI } & \multicolumn{4}{|c|}{ AOL } \\
\hline & coef & se & tstat & pval & coef & se & tstat & pval \\
\hline b0 & 0.1153 & 0.0415 & 2.7789 & 0.039 & 93.0543 & 37.957 & 2.4516 & 0.0578 \\
\hline b1 & -0.0677 & 0.0244 & -2.7734 & 0.0392 & 55.781 & 22.3319 & 2.4978 & 0.0546 \\
\hline b2 & 0.0395 & 0.0244 & 1.6194 & 0.1663 & 25.3215 & 22.3319 & 1.1339 & 0.3083 \\
\hline b3 & 0.0309 & 0.0244 & 1.2662 & 0.2612 & -14.7863 & 22.3319 & -0.6621 & 0.5372 \\
\hline b1b2 & 0.0466 & 0.0273 & 1.7084 & 0.1483 & 35.9486 & 24.9679 & 1.4398 & 0.2095 \\
\hline b1b3 & -0.0032 & 0.0273 & -0.1172 & 0.9112 & -2.8077 & 24.9679 & -0.1125 & 0.9148 \\
\hline $\mathrm{b} 2 \mathrm{~b} 3$ & -0.0102 & 0.0273 & -0.3728 & 0.7246 & 2.7659 & 24.9679 & 0.1108 & 0.9161 \\
\hline $\mathrm{b} 1^{2}$ & 0.1379 & 0.0481 & 2.8646 & 0.0352 & -28.0301 & 44.0392 & -0.6365 & 0.5525 \\
\hline $\mathrm{b} 2^{2}$ & -0.085 & 0.0481 & -1.7647 & 0.1379 & 38.5954 & 44.0392 & 0.8764 & 0.4209 \\
\hline $\mathrm{b} 3^{2}$ & 0.0307 & 0.0481 & 0.6388 & 0.5511 & -48.8444 & 44.0392 & -1.1091 & 0.3179 \\
\hline
\end{tabular}

Another advantage of the proposed approach is the development of a series of tools that allows the automation of the required calculations as well as the integration during the estimation process of the information related to decision maker attitude and risk aversion.

Despite the already cited benefits of the proposed RI, the latter can be further improved. In particular the possibility to create a bounded adimensional index on the basis of the current RI can facilitate the understanding of the robustness concept. Furthermore, the specific RI value can have a clear "absolute" meaning, which specifies how a production system is close to the theoretical maximum robustness, widening its use beyond the simple ranking of alternatives.

Due to the wide use of weighting factors and other decision maker specified parameters, another interesting perspective is constituted by the development and the integration of behavioural analysis tools to the robustness evaluation framework. In this way the obtained results can be used to elicit structured relationships between the behaviour characterisation and the specified parameter values.

In a broader perspective, the suitability of supplementary loss functions to be integrated in the multi-criteria performance measurement framework can also be studied in order to provide a comprehensive evaluation of the impact of a given SC configuration, for instance considering environmental and social performance measures.

\section{REFERENCES}

[1] CHRISTOPHER M., PECK H., 2004, "Building the Resilient Supply Chain", The International Journal of Logistics Management, 15, 2, 1-14.

[2] LONGO F., MASSEI M., 2008, "Advanced Supply chain Protection \& Integrated Decision support System", Proceedings of Second Asia International Conference on Modelling \& Simulation, 716-721.

[3] VLAJIC J.V., VAN DER VORST J.G.A.J., HENDRIX E.M.T., 2008, "Food supply chain network robustness, a literature review and research agenda", Proceedings of 8th International Conference on management in Agrifood Chains and Networks, 1-17.

[4] EROL O., HENRY D., SAUSER B., MANSOURI M., 2010, "A framework for investigation into extended enterprise resilience", Enterprise Information Systems, vol. 4 no. 2, 2010, p. 111 - 136.

[5] SÖRENSEN K., 2003, "A framework for robust and flexible optimisation using metaheuristics - with applications in supply chain design", Universiteit Antwerpen Faculteit Toegepaste Economische Wetenschappen, Ph.D. Dissertation

[6] KOUVELIS P., KURAWARWALA A.A., GUTIERREZ G.J., 1992, "Algorithms for robust single and multiple period layout planning for manufacturing systems", European Journal of Operational Research, 63, 287-303.

[7] ULUSOY G., UZSOY R., 1994, "A robust strategy approach to a strategic mobility problem", European Journal of Operational Research, 79, 266-276.

[8] GUTIERREZ G.J., KOUVELIS P., KURAWARWALA A.A., 1996, “A robustness approach to uncapacitated network design problems", European Journal of Operational Research, 94, 362-376

[9] ROY B., 1998, "A missing link in OR-DA Robustness analysis", Foundations of Computing and Decision Sciences, 23, 3, 141-160.

[10] POMEROL J.C., 2001, "Scenario development and practical decision making under uncertainty”, Decision Support Systems 31, 197-204.

[11] CHEN C-L., LEE W-C., 2004, “ Multi-objective optimization of multiechelon supply chain networks with uncertain product demands and prices", Computers and Chemical Engineering, 28, 1131-1144. 
[12] SEVAUX M., SÖRENSEN K., 2004, "A genetic algorithm for robust schedules in a one-machine environment with ready times and due dates", 4OR: A Quarterly Journal of Operations Research, 2, 2, 129-147.

[13] HITES R., DE SMET Y., RISSE N., SALAZAR-NEUMANN M., VINCKE P., 2006, "About the applicability of MCDA to some robustness problems", European Journal of Operational Research, 174, 1, 322-332.

[14] LEMPERT R.J., Groves D.G., Popper S.W., Bankes S.C., 2006, “A General, Analytic Method for Generating Robust Strategies and Narrative Scenarios", Management Science, 52, 4, 514-528.

[15] PIERREVAL H., DURIEUX-PARIS S., 2007, "Robust simulation with a base environmental scenario", European Journal of Operational Research, 182, 783-793.

[16] VOMMI V.B., SEETALA M.S.N., 2007, “A simple approach for robust economic design of control charts, Computers and Operations Research, 34, 7, 2001-2009.

[17] BEAMON B.M., 1999, "Measuring Supply Chain Performance", International journal of Operations and Production Management, 19, 275-292.

[18] CANETTA L., CHEIKHROUHOU N, and GLARDON R., 2013, "Modelling hybrid demand (e-commerce "+" tradi-tional) evolution: A scenario planning approach", International Journal of Production Economics, Volume 143, Issue 1, May 2013, Pages 95-108.

[19] BOUGHATtAS S., HARBI S., CANETTA L., 2016, "Sustainable Supply Chain design: towards a framework for measuring robustness", submitted to MOSIM 2016

[20] SPIRING F., 1991, "An alternative to Taguchi's loss function", Proceedings of 1991-ASQC Quality Congress Transactions- Milwaukee, 660-665.

[21] ANDERSON E.W., 1993, "The Antecedents and Consequences of Customer Satisfaction for Firms", Marketing Science, 12, 2, 125-143.

[22] LI M.H.C., 2003, "Quality Loss Functions for the Measurement of Service Quality", International Journal of Advanced Manufacturing Technology, 21, 29-37.

[23] MUKHERJEE A., NATH P., 2005, "An empirical assessment of comparative approaches to service quality measurement", Journal of Services Marketing, 19, 3, 174-184.

[24] BULT J.R., WITTINK D.R., 1996, "Estimating and validating asymmetric heterogeneous loss functions applied to health care fund raising”, International Journal of Research in Marketing, 13, 215-226.

[25] CHRISTOFFERSEN P., DIEBOLD F.X., 1997, "Optimal Prediction Under Asymmetric Loss", Econometric Theory, 13, 808-817.

[26] CLATWORTHY M.A., PEEL D.A., POPE P.F., 2005, "Are Analysts Loss Functions Asymmetric?", Working Paper. The Department of Economics, Lancaster University. http://ssrn.com/abstract $=875790$

[27] CHANG Y-C, HUNG W-L, 2007, "LINEX Loss Functions with Applications to Determining the Optimum Process Parameters", Quality \& Quantity, 41, 291-301.

[28] WEN D., LEVY M.S., 2001, "BLINEX: a bounded asymmetric loss function with application to Bayesian estimation", COMMUN. STATIST.-THEORY METH., 30, 1, 147-153.

[29] HACHEN C., 2003, "Double Speed Single Production Line: a new hybrid manufacturing planning and control system based on the optimal allocation of resources", EPFL Ph.D. Dissertation N. 2731.

[30] GRANGER C.W.J., 1969, "Prediction with a Generalized Cost of Error Function", Operational Research Quaterly 20, 2,199-207.

[31] CHENG T.C.E., OGUZ C., QI X.D., 1996, "Due-date assignment and single machine scheduling with compressible processing times", International Journal of Production Economics, 43, 29-35.

[32] CRONE S.F., 2002, "Training Artificial Neural Networks for time series prediction using asymmetric cost functions", Proceedings of the 9th International Conference on Neural Information Processing, 5, 23742380 .

[33] ELliOTT G., TIMMERMANN A.G., KOMUNJER I., 2003, "Estimating Loss Function Parameters". CEPR Discussion Paper No. 3821. Available at SSRN: http://ssrn.com/abstract $=404700$
[34] KAZANCIOGLU E., SAITOU K., 2004, "Multi-period robust capacity planning based on product and process simulations", Proceedings of the 2004 Winter Simulation Conference, 1781-1789.

[35] ANDERSSON J., KRUS P., 2001, "Metamodel representations for robustness assessment in multiobjective optimisation", Proceedings of the International Conference on Engineering Design (ICED 01 GLASGOW), 1-8.

[36] AL-AOMAR R., 2002, "A robust simulation-based multicriteria optimization methodology", Proceedings of the 2002 Winter Simulation Conference, 1931-1939.

[37] TAVANA M., 2003, "CROSS: A Multicriteria Group-Decision-Making Model for Evaluating and Prioritizing Advanced-Technology Projects at NASA", Interfaces INFORMS, 33, 3, 40-56.

[38] KO Y-H., KIM K-J., JUN C-H., 2005, “A New Loss Function-Based Method for Multiresponse Optimization”, Journal of Quality Technology, 37, 1, 50-59.

[39] BÜYÜKÖZKAN G., 2004, "Multi-criteria decision making for emarketplace selection", Internet Research, 14, 2, 139-154.

[40] JENSEN M.T, 2001, "Improving Robustness and Flexibility of Tardiness and Total Flowtime Job Shops using Robustness Measures", Applied Soft Computing, 1, 1, 35-52.

[41] KLEIJNEN, J.P.C., BETTONVIL B., PERSSON F., 2003, "Robust solutions for supply chain management: Simulation and risk analysis of the Ericsson case study. Working Paper (preprint: http://center.kub.nl/staff/kleijnen/papers.html)

[42] GIUSTOLISI O., MASTRORILLI M., "Optimal-Robust Design of Water Distribution Networks under Uncertain Scenario", www.casa.ucl.ac.uk/cupumecid_site/download/Giustolisi\&Mastrorilli.pd $\mathrm{f}$

[43] KLEIJNEN, J.P.C., GAURY E., 2003, "Short-term robustness of production management systems: A case study", European Journal of Operational Research, 148, 2, 452-465.

[44] KOUVELIS P., YU G., 1997, "Robust Discrete Optimization and Its Applications", Research monograph for Book Series on Non-Convex Optimization and Its Applications, Kluwer, Amsterdam.

[45] CANETTA L., 2009, "Prediction of hybrid (e-commerce and traditional) demand evolution and the assessment of its impact on production system robustness". EPFL thesis, $n^{\circ} 4382$. 